\title{
EDITORIAL
}

(O) ATRIAL FIBRILLATION

\section{Global collaboration needed for AF}

A lthough clinicians are always looking for objective ways to measure therapeutic success, most patients understandably consider quality of life to be the most important outcome of therapy. This viewpoint is particularly valid for patients with atrial fibrillation (AF), because few interventions have been shown to reduce mortality. When making a decision about which therapeutic strategy to use for a patient with AF, it is thus important to consider the patient's health-related quality of life (HRQoL) at baseline and how it might be affected by the various treatment options available. However, these considerations rely on the availability of an accurate means of testing AF-associated HRQoL. To date, clinicians have tended to use generic HRQoL questionnaires - most commonly the Short-Form health survey-36 items (SF-36; Stewart, A. L. et al. Medical Care 36, 724-735; 1988) — which might not detect subtle, but important, HRQoL changes in patients with AF. The field of AF needs a globally-employed, AF-specific HRQoL questionnaire so that we can make solid, but sensitive, comparisons between studies as well as well-informed clinical decisions.

Rate-control and rhythm-control interventions have repeatedly been demonstrated to improve HRQoL in patients with AF. However, trials comparing these two approaches to AF therapy have not shown that one is significantly better at improving HRQoL than the other. The inability to distinguish between the two approaches is likely to be related to the nonspecific questionnaires often used to test HRQoL in these studies, which are designed to measure general health and patient function, and can be influenced by concomitant disease. This influence is particularly strong for patients with AF because this condition predominantly affects the elderly, a group of people who tend to have multiple health problems. Moreover, these generic questionnaires do not take into account AF-specific symptoms and their effects on well-being. As a result, these generic questionnaires might not be sensitive enough to detect differences in improvement in AF-specific effects on function. Various AF symptom checklists have also been used; these instruments assess AF symptoms, but do not consider the impact on patient function or satisfaction-important components of HRQoL.

The use of an AF-specific HRQoL questionnaire might provide sufficient sensitivity to determine the type of therapeutic approach that will best improve HRQoL in the individual patient, and perhaps even to identify which approach is most appropriate across particular subgroups of patients with AF. However, unlike many other conditions, no simple, validated HRQoL questionnaire specific to AF has been used in large, multinational populations. Indeed, validation of AF-specific HRQoL questionnaires has only started to be reported in the past year or so, and only in single-country studies.

The Canadian Cardiovascular Society Severity of Atrial Fibrillation (CCS-SAF) scale identifies the presence of any of five symptoms - palpitation, dyspnea, dizziness or presyncope or syncope, chest pain, and weakness or fatiguedetermines whether these symptoms are associated with $\mathrm{AF}$, and then classifies the impact of AF-associated symptoms on the patient's function. Patients are classified on a scale that ranges from being asymptomatic with respect to $\mathrm{AF}$ (class 0 ) to having AF-attributable symptoms that severely affect the patient's HRQoL (class 4). This test has been validated against the SF-36 and quality-of-life components of the University of Toronto Atrial Fibrillation Severity Scale (AFSS; which only considers the symptom of palpitations) in 484 patients at five Canadian cardiology clinics (Dorian, P. et al. Circ. Arrhythm. Electrophysiol. 2, 218-224; 2009). No linear relationship was observed with the subjective measures of AF frequencies and duration on the AFSS; however, the investigators noted that the CCS-SAF scale was not designed to measure AF burden, just impact on HRQoL. Correlations between CCS-SAF class and the physical component summary of the SF-36 questionnaire at each study center were compared to assess the consistency of CCS-SAF class rating; no difference was found. Time taken to administer the questionnaire was not noted in the investigators' report. Nevertheless, the investigators believe that the CCS-SAF questionnaire represents a more simple and bedside-friendly option than the SF-36 and AFSS questionnaires.

The questionnaire for Quality of Life in AF patients (QLAF) - which consists of 22 questions related to palpitation, breathlessness, chest pain, dizziness, drugs, direct-current cardioversion, and ablation-is another AF-specific HRQoL questionnaire that has been validated against the SF-36 in 63 patients at a center in Brazil (Braganca, E. O. V. et al. Int. J. Cardiol. doi:10.1016/ j.ijcard.2009.03.087). The overall internal consistency and test-retest reliability measures were very high (for overall QLAF score, Cronbach a coefficient $=0.96$ for intraobserver and 0.98 for interobserver reproducibility, and intraclass correlation coefficient $[$ ICC $]=0.91$ for intraobserver and 0.95 for interobserver reproducibility) and were good for all individual domains, except for the interobserver reproducibility for the drugs domain (Cronbach $a$ coefficient $=0.58$ and $\mathrm{ICC}=0.41$ ). Administration of the QLAF questionnaire took one-third the time of its SF-36 counterpart-approximately $3 \mathrm{~min}$.
41 ...the international AF community must agree on a standard, easy-to-use, AF-specific HRQOL questionnaire 77

Valentin Fuster is the Editor-in-Chief and Bryony M. Mearns is the Editor of Nature Reviews Cardiology.

Competing interests The authors declare no competing interests. 
A disease-specific quality of life questionnaire for patients with AF (AF-QoL) has also been validated against the SF-36 in 417 patients at 29 Spanish centers (Arribas, R. et al. Europace doi:10.1093/europace/ eup421); this questionnaire contains seven questions related to psychology, eight questions related to physical activity, and three questions related to sexual activity in the past month. Approximately $5 \%$ of the AF-QoL questions related to sexual activity were not filled out by patients and, unlike the scores for the other two domains as well as the overall scores, the scores for this domain did not discriminate between patients with AF and patients with prior myocardial infarction. The internal consistency was high (Cronbach $\alpha$ coefficient $=0.92$ for overall score and $>0.80$ in each domain), as was the test-retest reliability (ICC $>0.80$ for overall score and for each domain). Self-administration of the AF-QoL took a median of $10 \mathrm{~min}$.

Finally, another new self-administered instrument that consists of six questions related to dyspnea at rest and during exertion as well as AF-related limitations in daily life, discomfort, fatigue, and worry or anxiety (the
AF6 instrument) has been validated against the SF-36 and the Toronto Symptoms Check List in 111 patients at a single center in Sweden (Härdén, M. et al. Health Qual. Life Outcomes 7, 65; 2009). The internal consistency was high (Cronbach a coefficient $=0.81$ ) and the test-retest reliability was high for the first three questions (ICC of 0.82-0.93), but low for the last three questions (ICC of 0.04-0.39). Although no exact time taken to administer the questionnaire was noted in their report on validity, one of the investigators' requirements when designing the questionnaire was that it should be simple for patients to complete within a $20 \mathrm{~min}$ patient visit.

We need to make large-scale, multinational testing of a simple AF-specific HRQoL questionnaire a priority. To ensure that the debate progresses on whether rate or rhythm control is the better therapeutic strategy for patients with AF, and to enable us to make well-informed clinical decisions for individual patients with AF, the international AF community must agree on a standard, easy-to-use, AF-specific HRQoL questionnaire.

doi:10.1038/nrcardio.2010.12 